Hakam H Sabah BDS, MSc (Lec.)

\section{Effect of Enamel Resin-Removal Methods on Tensile Bond Strength of Rebonded New Brackets}

\author{
Dept of Pedod, Orthod, and Prev Dentistry
}

College of Dentistry, University of Mosul

\begin{abstract}
الخحلاصة
الأهداف: تحدف الدراسة الى تعيينَ تأثير الطرائقِ المختلفة لإزالة الراتنج على قوّة شد الترابط (TBS) للحاصرات الجديدة و المعاد لصقها و المقارنة بين الطرائقِ

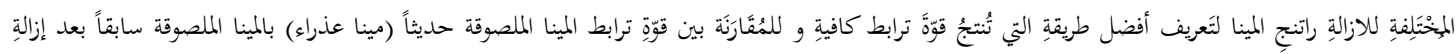

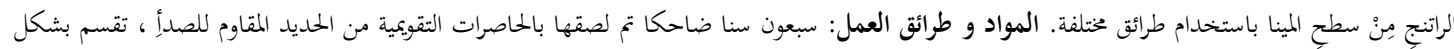

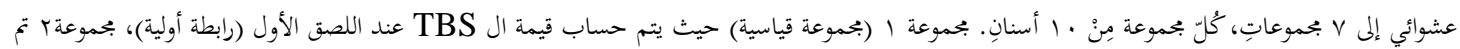

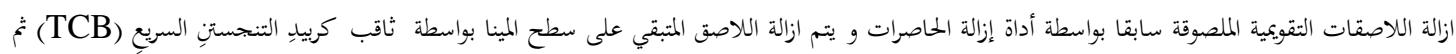

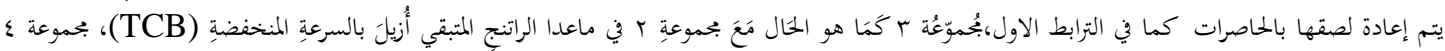

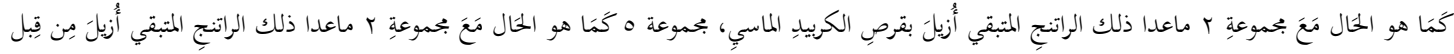

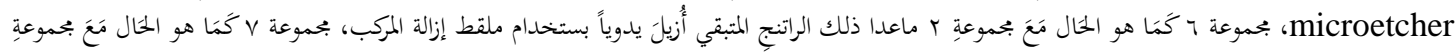

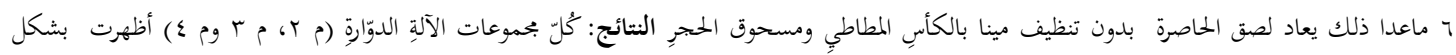

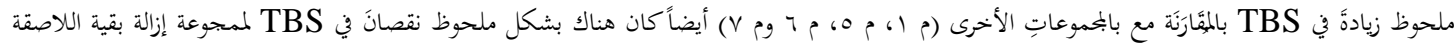

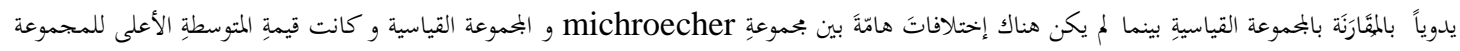

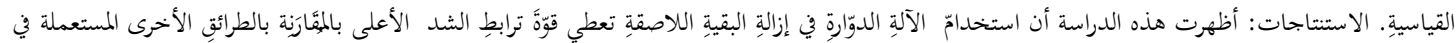

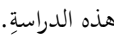
ABSTRACT
Aims: The objective of this study is to determine (1) the effect of different resin-removal methods on
tensile bond strength (TBS) of rebonded new brackets (2) compare various methods of enamel resin-
removal to define the best method that produces adequate bond strength (3) to compare the bond
strength of newly bonded enamel (virgin enamel) with previously bonded enamel after resin removal
from the enamel surface with different methods. Materials and Methods: Seventy human premolars
were bonded with stainless steel new brackets, then the teeth were randomly assigned in to 7 groups,
each group of 10 teeth. Group1 (control group) where TBS at the first debonding was evaluated (initial
bond). Group2 brackets were debonded by bracket removal plier and the remnant adhesive removed
by high-speed tungsten carbide bur (TCB) and rebonded with brackets as in initial bond, then the TBS
were tested. Group3 as with group2 except that the remaining resin were removed by low-speed TCB .
Group 4 as with group2 except that the remaining resin were removed by Diamond carbide Disk
Group 5 as with group2 except that the remaining resin were removed by a microetcher, Group 6 as
with group 2 except that the remaining resin were removed manually by composite removing pliers,
Group 7 as with group6 except that rebonding bracket without cleaning enamel with rubber cup and
pumice. Results: All rotary instrument groups (G2, G3 and G4) showed significant increase in the
TBS in comparing with the other groups (G1, G5, G6 and G7) also there were significant decrease in
the TBS of the manually removal of adhesive remnant groups in comparing with control group, while
there were no significant differences between microetcher group and control group with greater mean
value for control group. Conclusions: The outcomes of this study showed that the use of the rotary
instrument in the removal of adhesive remnant gives the highest tensile bond strength in compared with
the other methods used in this study .
Key words: Adhesive, Remnant index, Bond strength, Orthodontic brackets. Sabah HH. Effect of Enamel Resin-Removal Methods on Tensile Bond Strength of Rebonded New Brackets. Al-Rafidain Dent J. 2013; 13(1): 176-183.

Received: 6/2/2012 Sent to Referees: 12/2/2012

Accepted for Publication: 28/3/2012

\section{INTRODUCTION}

It has been shown that, in spite of being transient, bonding between the bracket base and the enamel surface must be strong enough to withstand stresses and shear force. ${ }^{(1)}$ The unplanned and 
accidental dislodgment of an orthodontic brackets is a common occurrence among orthodontic patients. Various factors can contribute to the likelihood of a bond failure, including operator technique, patient behavior, variation in the enamel surface, occlusal trauma and brackets properties, ${ }^{(2)}$ Improper orthodontic bracket position or bonding failure may necessitate the bracket removal and rebonding to establish a correct bracket position during the treatment to take full advantages of arch wire slot values and sliding mechanics. ${ }^{(3)}$ Before rebonding an orthodontic bracket, the following elements should be consider: reconditioning of the enamel surface, the use of new brackets or the original brackets, and the bonding system to be used. ${ }^{(4)}$ The search for an efficient and safe method of adhesive resin removal after debonding has resulted in the introduction of a wide array of instrument and procedures. $^{(5)}$ These include manual removal with the use of a scaler or bandremoving plier, ${ }^{(6)} 12$ fluted finishing bur in a high-speed dental hand piece under dry condition, ${ }^{(7)}$ various shapes of tungsten-carbide bur with low-or high speed hand piece, ${ }^{(8)}$ sof-lex discs, and special composite finishing systems with zirconia paste or slurry pumice as well as ultrasonic applications, ${ }^{(9)}$ microetcher by Aluminum oxide air-abrasion in spit of its increasingly widespread use for recycling purposes and improve bracket bonding to restored teeth as will as to prepare the enamel surface, ${ }^{(10)}$ although there is a notable lack of a universally approved protocol for this potentially litigious treatment stage. ${ }^{(11)}$

Damage of enamel can be attributed to cleaning with abrasive before etching, acid etching, enamel fractures cause by forcibly removing brackets, or mechanical removal of remaining composite with rotary instrument. ${ }^{(12)}$

Along with the introduction of novel methods, the armamentarium of conventional instruments has been fortified by the introduction of specially designed burs which are less aggressive to enamel. ${ }^{(13)}$ To remove residual material, tungsten carbide burs are preferable to other methods, such as diamond burs, sandpaper disks, or rubber wheel. Water cooling is recommended when bulk material is removed at high speed to avoid pulpal damage. When remnants of adhesive are removed at low speeds, better contrast between the adhesive and enamel is obtained without water cooling. ${ }^{(14)}$

The bond strength of a rebounded bracket has been reported to exceed the minimum force requirement of 6 to $8 \mathrm{Mpa}$ ${ }^{(4)}$ however, there is no consensus on how rebond strength compares with original bond strength, some authors have reported that rebond strength is lower, ${ }^{(15,16)}$ while others have reported that it is either comparable to or greater than that of the original bond..$^{(4,9,17,18)}$ Thus, it is important to understand what to expect when a tooth is rebounded one or more times, because the literature provides contradictory findings regarding the shear bond strength of rebounded attachments. ${ }^{(9)}$

The bond strength of attachments must be sufficient to withstand functional forces, but at a level to allow bracket debonding without causing damage to the enamel, which may occur when bond strength exceeds $14 \mathrm{Mpa}^{(19)}$

\section{MATERIALS AND METHODS}

Seventy healthy, caries free and intact human maxillary premolars extracted for orthodontic reasons within the previous 40 days from testing were used in the study, the extracted teeth were washed free of adherent blood and clean from the remnants of tissue by using tap water and fine brush. The teeth stored initially in $70 \%$ ethyl alcohol to prevent bacterial growth ; then the samples were kept in sterilized normal saline at room temperature to prevent dehydration until the start of the experiments. ${ }^{(20)}$

\section{Specimen preparation:}

The crowns of the teeth were separated from the roots at cervical line through using a diamond bur; and an under cut was made in pulp chamber to ensure remaining the crown in its position during measuring tensile bond strength. ${ }^{(21)}$

Glass slide is painted with separating medium (Vaseline) around the stick wax where crown is fixed at palatal surface in such away that the middle third of buccal surface was oriented to be parallel with 
analyzing rod of surveyor. The plastic ring of $1.5 \mathrm{~cm}$ in length and $1.5 \mathrm{~cm}$ in diameter is positioned around the fixed tooth in such away that the buccal surface of the crown portion of the tooth is protruded, then the powder and liquid of cold -cure acrylic resin are mixed and poured around the crown of the tooth, the crown were embedded in acrylic blocks leaving only the buccal surface of the crown exposed and left for setting for one hour after that, all samples were immersed in normal saline to prevent dehydration of the teeth. ${ }^{(22)}$

\section{Initial bond (sample preparation)}

All the teeth used in this study were bonded with stainless steel upper premolar brackets (ULTRA-MINITRIM, Standard Edgewise.018,No.790-010-80,Dentaurum) with the use of composite resin made specifically for orthodontic (BIOFIX Light Cured, Brackets fixing Adhesive, Biodinamica Europa).

Procedures of bonding were according to manufacturer instruction. The buccal surface of each tooth was cleaned, Polished, with non-fluoridated pumice and water slurry in a rubber cup attached to a low speed hand piece for 10 seconds, following which, the teeth were washed with the water spray for 30 seconds, and dried for 30 seconds then, the enamel was etched with 37\% Orthophosphoric AcidEtch tg blue gel (Gel $2.5 \mathrm{ml}$, Technical and General, UK, Lonaon W6 OJD, code:EE $4 \mathrm{~K})$ for 60 seconds and rinsed with water for 30 seconds then the teeth were dried with an oil free stream of air for 20 seconds.

The buccal enamel surface of the etched tooth appears chalky white in color, then the bracket was bonded to the tooth according to the manufacture instructions by apply a thin layer of Biofix light cured on the bracket base; immediately after that carefully placed the bracket in the middle third of the buccal surface and parallel to the long axis and adjusted to the correct position using a clamping tweezers. Press slightly to remain a thin layer of approximately $0.5 \mathrm{~mm}$ and any excess resin squeeze out from the edges carefully removed with dental probe without distributing the seat bracket, so that not overlap the base. The composite was lightcured for 40 seconds (10 seconds each side of bracket). The adhesive was allowed to set for 10 minutes before all samples were stored in distal water at room temperature.

The teeth randomly assigned in to 7 groups, each group of 10 teeth as follows:

Group 1: Control group, where tensile bond strength (TBS) at the first debonding was evaluated by the universal test machine (Zweigle models 73, made in Belgium) and adhesive on the bracket base were evaluated by modified ARI (Adhesive Remnant Index). ${ }^{(9)}$

Group 2: The brackets were debonded by bracket removal plier in occlusogingival direction with slight shear force (GAC International, Inc, Bohemia, NY). The remnant adhesive resin on the enamel surface were removed and reconditioned by high-speed tungsten carbide bur (TCB) (Dentsply Maillefer, USA, iso 012,314) with air cooling , cleaned with rubber cup and pumice, and Rebonded with new bracket as in initial bond, then debonded by the universal test machine and the TBS determined. The ARI scores were evaluated after that.

Group 3: The brackets and enamel surfaces were treated as with group 2, excepted that the remaining resin were removed by low-speed TCB (Dentsply Maillefer, USA, iso 012,197) with air cooling.

Group 4: The brackets and enamel surfaces were treated as with group 2, excepted that the remaining resin were removed by Diamond carbide discs (SPDENT, GmbH ,0123) with air cooling.

Group 5: The brackets and enamel surfaces were treated as with group 2, excepted that the remaining resin were removed by a microetcher model II (Danville Engineering Co.,USA). The tooth surface were held approximately 5 $\mathrm{mm}$ from tip of the microetcher (figure 1A) and cleaned with $50-\mu \mathrm{m}$ aluminum oxide particle under an enclosed ventilated hood. $^{(9)}$ 

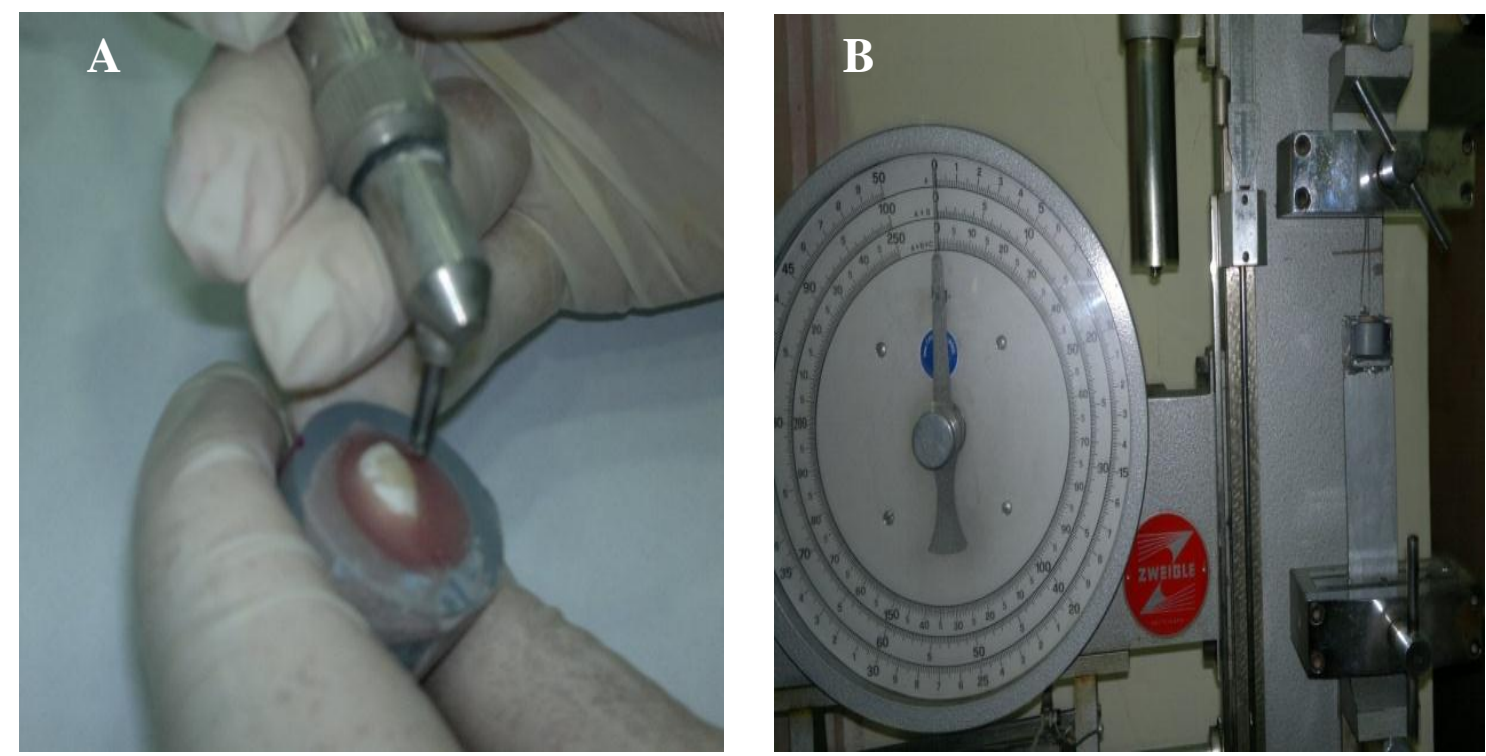

Figure (1): A; photographs showing the method used in removal of adhesive remnant by microecher $\mathrm{B}$; photographs showing the set-up for the tensile stress determination (machine, specimen and specimen container).

Group 6: The brackets and enamel surfaces were treated as with group 2, excepted that the remaining resin were removed manually by composite removing plier (Ortho Organizers,USA, 201-105 A1).

Group 7: The brackets and enamel surfaces were treated as with group 2, excepted that the remaining resin were removed by composite removing plier (Ortho Organizers,USA, 201-105 A1) alone without cleaning with rubber cup and pumice.

In all groups the removal of the composite was considered complete when tooth surface seemed smooth and free of composite to the naked eye under the light of an operatory lamp. ${ }^{(23)}$

\section{Tensile Bond Strength test (TBS):}

The TBS of brackets in all groups were tested by using the universal testing machine in textile factory in Mosul city (Figure 1-B). The set-up used has been described previously. ${ }^{(24)}$ A $.017 \times .025$-in stainless steel rectangular arch wire(performed ligature wires,751-001; Dentaurum), ${ }^{(25)}$ bent in a U-form and tied with a harness ligature to the bracket, the specimen with the specimen container (have been specially made for this study) were fixed in the lower jaw of the tensile testing machine, while the free ends of the wire were attached on the upper jaw of the tensile testing machine (Figure 1-B). A hinge in the connecting piece, together with the round wire, made vertical alignment of the specimen in the pre-test phase possible. Vertical alignment of the specimen is necessary for homogeneous stress distribution during the test over the specimen,${ }^{(26)}$ in tensile mode the upper jaw remained fixed and the lower jaw moved away from the upper jaw at the required speed $0.5 \mathrm{~mm} / \mathrm{sec}$.

At the moment when bracket tinseled from the tooth by force applied by testing machine, the reading was taken from the gage attach to the upper jaw.

\section{Modified adhesive remnant index} (ARI):

After TBS test all samples were examined under stereomicroscope at a magnification of $\times 10$ to determine the amount of composite resin remaining in the bracket bases using the modified adhesive remnant index (ARI). The modified ARI was used to determine the nature of bond failure. The modified ARI scale has a range of 5 to $0(5=100 \%$ of adhesive left $\mathrm{n}$ the bracket base; $4=100$ $75 \%$ adhesive left on the bracket base ; $3=$ $75-50 \%$ adhesive left on the bracket base; $2=50-25 \%$ adhesive left on the bracket base $; 1=$ less than $25 \%$ of adhesive left on 
the bracket base; $0=$ no adhesive left on the bracket base. ${ }^{(9)}$

\section{RESULTS}

The descriptive analysis (minimum, maximum, mean value, and SD) of tensile bond strength for all groups, are given in Table (1).

It can be noticed, that the mean TBS of high speed group (G2) is the highest (61.05), while the manually resin removal with polishing (G6) the lowest (9.72).

Table (1): Descriptive Statistics Demonstrating the Effect of Groups on Tensile Bond Strength of Rebonded new Brackets.

\begin{tabular}{ccccccc}
\hline Groups & No. & Mean* & Minimum & Maximum & SD & SD \\
\hline Control & 10 & 44.99 & 40.30 & 50.96 & 4.25 & 1.34 \\
High speed & 10 & 61.05 & 60.17 & 62.32 & .566 & .179 \\
Low speed & 10 & 49.25 & 40.67 & 51.45 & 3.62 & 1.14 \\
Disk & 10 & 51.09 & 40.67 & 60.76 & 5.33 & 1.68 \\
Microetcher & 10 & 44 & 37.30 & 50.18 & 3.69 & 1.16 \\
Manual with polishing & 10 & 38.03 & 30.87 & 41.74 & 4.58 & 1.44 \\
Manual without polish & 10 & 38.49 & 30.78 & 40.77 & 4.11 & 1.30
\end{tabular}

* Mean Measurement in N (Newton); SD: standard deviation; SE: standard error of mean.

The analysis of variance (ANOVA) for all groups showed significant differences $(p<0.001)$ among them as illustrated in Tables (2). The results of Duncan Multiple Analysis Range Test (Tables 2) for manually removal of old adhesive groups (G6 and G7)) showed significant decrease on TBS mean in comparison with control (G1) and all the other groups at $(p \leq 0.05)$, while micretched group showed no significant differences in comparison with control group but showed significantly decrease in compared with rotary instruments groups (G2,G3 and G4) .

Also the result of Duncan Multiple Analysis Range Test showed that high speed group (G2) had the significant increase on TBS mean in comparison with all groups at $(p \leq 0.05)$, also low speed and disk groups (G3 and G4) showed significant increase in tensile bond strength in compare with control group at $(p \leq 0.05)$.

T able (2): ANOVA and Duncan's Among all Groups for Determining the Effects of different methods used for adhesive remnant removal in the tensile Bond Strength of rebounded new

Brackets.

\begin{tabular}{ccccccc}
\hline & & Sum of square & df & Mean square & F- Value & $p$ \\
\hline \multirow{5}{*}{ ANOVA } & $\begin{array}{c}\text { Between } \\
\text { groups }\end{array}$ & 3844.7 & 6 & 640.78 & & \\
& $\begin{array}{l}\text { Within } \\
\text { groups }\end{array}$ & 1003.4 & 63 & 15.928 & 40.22 & $p<0.001$ \\
& Total & 4848.2 & 69 & & & \\
& Groups & Mean* \pm SE & & & \\
\hline \multirow{5}{*}{ Duncan } & G2 & $61 \pm 0.17$ & Duncan Groups** & \\
& G4 & $51 \pm 1.68$ & A & \\
& G3 & $49.2 \pm 1.14$ & B & \\
& G1 & $44.9 \pm 1.34$ & C & \\
& G5 & $44 \pm 1.16$ & C & \\
& G7 & $38.4 \pm 1.30$ & D & \\
& G6 & $38 \pm 1.44$ & D & \\
$*$ Measurement in N (Newton); ** Different letters mean significant difference $(p<0.05)$. &
\end{tabular}


Table (3) shows the descriptive analysis (minimum, maximum, mean value, and SD) of modified ARI for all groups. It can be noticed, that the mean of ARI group (G6) is the highest (3.60), while the ARI of (G2) the lowest (9.72), the analysis of variance (ANOVA) for all groups showed significant differences $(p<0.001)$ among them as illustrated in Tables (4). The result of Duncan Multiple
Analysis Range Test (Tables 4) showed that the rotary instruments groups (G2,G3 and G4) had significantly decrease in the modified ARI score in compare with all the others groups, also there are no significant differences in the modified ARI between manual adhesive removal Groups (G6 and G7) and microetcher group (G5) in compared with control group and group (G1).

Table (3): Descriptive Statistics Demonstrating the Effect of Groups on modified ARI scores .

\begin{tabular}{ccccccc}
\hline Groups & No. & Mean* & Minimum & Maximum & SD & SE \\
\hline Control & 10 & 3.30 & 3 & 4 & .483 & .152 \\
High speed & 10 & 1.50 & 1 & 2 & .527 & .166 \\
Low speed & 10 & 1.90 & 1 & 3 & .567 & .179 \\
Disk & 10 & 1.80 & 1 & 3 & .632 & .200 \\
Microetcher & 10 & 3.40 & 2 & 5 & .843 & .266 \\
Manual with polishing & 10 & 3.60 & 3 & 4 & .516 & .163 \\
Manual without polish & 10 & 3.50 & 3 & 4 & .527 & .166 \\
\hline
\end{tabular}

* Mean Measurement by score=0-5; SD: standard deviation; SE: standard error of mean.

Table (4): ANOVA and Duncan's Among all Groups for Determining the different in the modified ARI scores.

\begin{tabular}{|c|c|c|c|c|c|c|}
\hline & & Sum of square & df & Mean square & F-Value & $p$-value \\
\hline \multirow{3}{*}{ ANOVA } & $\begin{array}{c}\text { Between } \\
\text { groups }\end{array}$ & 51.886 & 6 & 8.648 & \multirow{3}{*}{24.321} & \multirow{3}{*}{$p<0.001$} \\
\hline & $\begin{array}{l}\text { Within } \\
\text { groups }\end{array}$ & 22.400 & 63 & .356 & & \\
\hline & Total & 74.286 & 69 & & & \\
\hline & Groups & Mean* \pm SE & \multicolumn{4}{|c|}{ Duncan Groups** } \\
\hline \multirow{7}{*}{ Duncan } & G1 & $3.30 \pm .152$ & \multicolumn{4}{|c|}{ A } \\
\hline & G5 & $3.40 \pm 2.66$ & \multicolumn{4}{|c|}{ A } \\
\hline & G6 & $3.60 \pm .163$ & \multicolumn{4}{|c|}{ A } \\
\hline & G7 & $3.50 \pm .166$ & \multicolumn{4}{|c|}{ A } \\
\hline & G3 & $1.90 \pm .179$ & \multicolumn{4}{|c|}{ B } \\
\hline & G4 & $1.80 \pm .200$ & \multicolumn{4}{|c|}{ B } \\
\hline & G2 & $1.5 \pm .166$ & \multicolumn{4}{|c|}{ B } \\
\hline
\end{tabular}

* Measurement by score $=1-5$; $* *$ Different Litters Mean significant difference $(p \leq 0.05)$.

\section{DISCUSSION}

In the present study, only 1 type of cement and bracket where used to ensure that the any significant variations in TBS were clearly attributable to methods used in adhesive remnant removal.

The results of this study showed that removal of adhesive remnant using high speed rotary instrument (high speed TCB ) not only provided TBS comparable to that of the initial TBS (control G), but also provided higher TBS values than the initial TBS. While removal of adhesive remnant by using less aggressive methods of rotary instrument (Low speed TCB and Diamond carbide disk) also result in higher TBS compared with initial bond strength. This result is in agreement with Mui et al.,${ }^{(4)}$ and Neslihan et al., ${ }^{(9)}$ and Behnam et al. ${ }^{(18)}$ and not agree with regan et al., ${ }^{(16)}$ wright and powers ${ }^{(27)}$ they founded that rebounded new brackets demonstrate a small, but statistically significant fall in bond strength in 
compared with initial bond strength.

The increased rebond strength with the rotary instrument groups may be due to an increase in enamel roughness after resin removal and an increase in the mechanical retention. ${ }^{(9)}$ However, the results of this study showed statistically significantly decrease in the TBS for both low speed TCB and Disk in compare with high speed TCB this result may be due to that TCB used at high speed, can cause damage to enamel because they are harder than the enamel, ${ }^{(6)}$ while TCB and Diamond carbide disk at low speed produced the finest scratch pattern with less enamel loss of $7.4 \mu \mathrm{m}$. $^{(23)}$

It has been suggested that increase enamel roughness following resin removal may effectively be diminished by masticatory loads and the friction developed between the enamel surface and various hard foods. ${ }^{(11)}$

Also the results of this study showed statistically significant decrease in the TBS of manual remnant removal groups (G6 and G7) in compared with control groups. This finding clearly due to old adhesive remnant in the enamel surface after resin removal, this result is in agreement with Mui et al., ${ }^{(4)}$ and NESLIHAN ETAL ${ }^{(9)}$ and not agree with regan et al., ${ }^{(16)}$ how found that there was no difference in tensile bond strength following removal of adhesive from enamel surface with either a hand scaler or tungsten-carbide bur prior to rebonding.

Also The result of this study showed no statistically difference between the microetcher group and control groups .This result could be due to the fact that the microetcher cause irreversible loss of enamel by removal of both organic and inorganic components of the enamel matrix $^{(27)}$ and this may lead to different surface patterns after acid etch, Neslihan et al., ${ }^{(9)}$ founded under SEM, the enamel seemed smooth at $300 \times$, but at $1500 \times$ magnification revealed deep pit after resin remnant removal by microetcher, which may be the possible cause of the lower rebond strength found in this group in compared with rotary instrument groups.

The modified ARI in this study showed that there is significant increase in the ARI score for rotary instrument groups in compare with manual groups and control group. The high modified ARI score found for manual groups and control group indicates that the bond between the bracket and adhesive was much stronger than the adhesive-enamel surface, while the decrease in the ARI score found for the rotary instrument groups indicates that the bond between the enamel surface and adhesive was much stronger than the adhesive-bracket and the bracket-adhesive bond forms the weakest point. This result improving and support the result obtain from the TBS test.

\section{CONCLUSIONS}

The tensile rebond strengths after resin removal with high-speed TCB, low-speed TCB, and Diamond carbide discs are higher than the initial, microetcher and manual removal of resin remnant. This study supports the used of low speed TCB and diamonds disk in the removal of old adhesive remnant for rebond new bracket because they are less aggressive to enamel surface, reasonable application and gives high TBS in compare with the initial bond in spit of this the High speed TCB gives the higher TBS in compared with all other Groups but it is more aggressive than other groups. The bond strength of rebond new bracket could be less or more than the intial bond strength depend on the methods use for adhesive remnant removal.

\section{REFERENCES}

1. Stenyo W, Simonides C, Darcy F. Shear bond strength of new and recycled brackets to enamel. Braz Dent J. 2006; 17(1): 103-440.

2. Stephen D, Ross S. Comparison of in vivo and in vitro shear bond strength. Am J Orthod Dentofacial Orthop. 2003; 123: 29.

3. Gaffey P, Major P, Glover K, Grace M, Koehler J. Shear/peel bond strength of repositioned ceramic brackets. Angle Orthod. 1995; 65(5): 351-357.

4. Mui B, Rossouw P, Kulkarni G. Optimization of a procedure for rebonding dislodged orthodontic brackets. Angle Orthod. 1999; 69(3): 276-281.

5. Pus M, Way D. Enamel loss due 
orthodontic bonding with filed and unfiled resins using various clean up techniques. Am J Orthod.1980; 77: 269-283.

6. Rouleau B, Marshall G, Cooley R. Enamel surface evaluation after clinical treatment and removal of orthodontic brackets. Am J Orthod. 1982; 81: 423-426.

7. James K, Robert N, Jane R: The effect of microetching on the bond strength of metal bracket when bonded to previously bonded teeth. Am J Orthod Dentofacial Orthop. 1998; Oct: 452-460.

8. Campbell P: Enamel surfaces after orthodontic bracket debonding. Angle Orthod. 1995; 65: 103-110.

9. Neslihan E, Ayca A, Alev C, Erdem K: Effect of Resin-removal Methods on Enamel and Shear Bond Strength of Rebonded brackets. Angle Orthod. 2006; 76: 314-321.

10. Millet D, Mccabe J, Gordon P: The role of sandblasting on the retention of bonded metallic brackets applied with glass ionomer cement. Brit J Orthod. 1993; 201: 117-122.

11.Eliades T, Gioka C, Eliades G, Makou M: Enamel surface roughness following debonding using two resins grinding methods. Eur J Othod. 2004; 26: 333-338.

12.Hosein I, Scherriff M, Ireland A. Enamel loss during bonding, debonding and cleanup with use of self-etching primer. Am J Orthod Dentofacial Orthop. 2004; 126: 717-72 4.

13.Radlanski R. A new carbide finishing bur for bracket debonding. $J$ Orofacial Orthop. 2001; 62: 296-304 .

14.Graber T, EliadesT, Athanasiou A. Risk management in Orthodontics.Quintessence publishing Co. Inc. 2004; Pp: 40-41.

15. Wright $\mathrm{G}$, hatibovic-kofman $\mathrm{S}$, Milenaar $\mathrm{D}$, Bravermanl. The safety and efficacy of treatment with air abrasion technology. Int J Paed Dent.1999; 9: 133-140.

16.Regan D,Lemasney B, Vannoort: The tensile bond strength of new and rebounded stainless steel orthodontic brackets. Eur J Orthod.1993; 15: 125-153.

17.Leas T, Hondrum S. The effect of rebonding on the shear bond strength of orthodontic brackets-a comparison of two clinical techniques. Am $J$ Orthod Dentofacial Orthop. 1993; 103: 200-201.
18.Behnam K, Saeed N, Sahar N, Vahid R : Assessing the effects of three resin removal methods and bracket sandblasting on shear bond strength of metallic orthodontic brackets and enamel surface. Orthod Waves. 2011; 70:27-38.

19. Pickett K, Sadowsky P, Jacobson A, Lacefield W: Orthodontic in vivo bond strength: comparison with in vitro results. Angle Orthod. 2001;71:141-148.

20.Chung K, Hsu B and Hsieh T: effect of sandblasting on the bond strength of the bondable molar tube bracket. J Oral Rehabil. 2001; 28:418-424.

21.Omar H, Hussain A, Amer A. Recycling of Orthodontic Metal Brackets. MSc thesis. College of Dentistry, Mosul University. 2005.

22.Tecco S, Traini T, caputi S, Luca V, D'Attilio M. A new one-step dental flow able composite for orthodontic use: an in vitro bond strength study. Angle Orthod. 2005; 75(4): 672-677.

23. Van Waes H, Matter T, Krejci I. Three dimensional- measurement of enamel loss caused by bonding and debonding of orthodontic bracket. Am J Orthod Dentofacial Orthop. 1997; 112: 666-669.

24.Tjalling JA, Cornelis JK, De Gee JA, Birte PA, Albert JF. The influence of accelerating the setting rate by ultrasound or heat on the bond strength of glass ionomers used as orthodontic bracket cements. Eur J Orthod. 2005; 27: 472-476.

25.Ilken K, Senay C, Kivanc A. Tensile bond strength of ceramic orthodontic brackets bonded to porcelain surface. Am J Orthod Dentofacial Orthop. 2001; 119 (6): 17-20.

26.Tjalling J.A, Cornelis J.K, Birte P.A, Albert J.F: The influence of different bracket base surfaces on tensile and shear bond strength. Eur J Orthod. 2008;30: 490-494.

27. Wright W, Powers J: In vitro tensile bond strength of reconditioned brackets. Am J Orthod. 1985;87:247-252

28.Olsen ME, Bishara SE, Damon P, Jakobsen JR. Comparison of shear bond strength and surface structure between conventional acid etching and air-abrasion of human enamel. Am $J$ Orthod Dentofacial Orthop. 1997; 112: 502-506. 